\title{
The Effects of Intermolecular Energy Transfers on a Closed Power Cycle's Efficiency
}

\author{
David Van Den Einde ${ }^{1}$ \\ ${ }^{1} 1284170^{\text {th st }}$, Bejou, Minnesota 56516, USA \\ Correspondence: David Van Den Einde, 1284 170 ${ }^{\text {th st }}$, Bejou, Minnesota 56516, USA. E-mail: dmvde@arvig.net
}

Received: November 11, 2017

Accepted: December 20, 2017

Online Published: January 2, 2018

doi:10.5539/apr.v10n1p5

URL: https://doi.org/10.5539/apr.v10n1p5

\begin{abstract}
The efficiency of the power cycle described in this paper is affected by the transfer of energy between molecular potential and translational during the expansion and contraction of its working fluid. How the thermodynamic interrelationships between the temperature, volume changes, and changes in the molecular mean free paths determine this energy shift and their affects on cycle efficiency is discussed.
\end{abstract}

Keywords: Power cycle, Second Law, Carnot efficiency, thermodynamics, molecular mean free path

\section{Introduction}

This paper discusses the effect on a power cycle's efficiency caused by the transfer of energy from molecular deformation or potential energy to molecular translation and back during the working fluid's volume changes. The closed condensing non flow through transcritical cycle depicted in Fig. 1 uses as its working fluid a low boiling point solvent and a solid solute that yields a positive excess enthalpy of solution reaction in the solvent's liquid state at the cycle's T2 near point A. The solute experiences retrograde solubility in the solvent's supercritical region near the cycle's point $C$. The heat energy used to satisfy the positive excess enthalpy requirement at the cycle's T2 is derived either from energy not exhausted by bond formation during solvent condensation or from an outside source near T2. This energy is elevated to near the cycle's T1 during the solute's recrystallization.

Heat is input at constant volume from A to B from ascending thermal reservoirs one degree apart. Heat is input from $\mathrm{B}$ to $\mathrm{C}$ from ascending thermal reservoirs one degree apart at a constant $12 \mathrm{MPa}$. Adiabatic expansion occurs from C to D. Heat is exhausted isothermally and isobarically between D and A. Staging the heat input to the cycle is for purposes of discussion only, though the transvers flow heat exchanger used in a permutation of this cycle in Van Den Einde (2016a) approaches the effectiveness of the process.

By calculating the Carnot T1-T2/T1 heat to work conversion potential of the heat input from each reservoir and summing these work values, it is determined that that value equals the cycle's actual work output determined from the total Q1 heat input minus the total Q2 heat exhausted. Even though the portion of heat input energy that goes to bond stretching would appear to be a wasted throughput, staging the heat input causes the cycle's Q efficiency to match the $\mathrm{T}$ efficiency of the staged input heat. Any wasted throughput should cause the Q efficiency/T efficiency ratio to drop below 1.00. This paper explains how the shift of energy from molecular deformation during collision to molecular translation during changes in volume from the heat input and the inverse shift during fluid condensation at the common $\mathrm{T} 2$ establishes the $\mathrm{Q}$ efficiency/T efficiency ratio near 1.00. The $\mathrm{Q} / \mathrm{T}$ ratios of 0.995 to 1.005 found for the compounds in REFPROP (National Institute of Standards and Technology, Boulder, CO, 2007), that have data uncertainty in the plus or minus $1 \%$ range serve as empirical confirmation of the conclusions reached in this discussion (Van Den Einde, 2017).

\section{Discussion}

The working fluid's enthalpy change from B to C caused by the heat input from each ascending thermal reservoir is partitioned three ways. At each temperature some of the input energy goes to external work, some goes to the molecular freedoms, and some goes to bond stretching. The portion going to external work is transferred directly from heat to work by the molecules' translational freedom during isobaric expansion from $\mathrm{B}$ to $\mathrm{C}$ at the instantaneous temperature that it enters the cycle. The translational freedom both transfers energy to work by its increased velocity and along with the internal freedoms, simultaneously stores energy with increases 
in temperature. Because the heat input occurs at one degree ascending increments, the energy transfer to work can, for discussion, be treated as having occurred isothermally. This energy transfers is analogous to the isothermal heat to work of the ideal gas Carnot cycle. The portion of the input energy that goes directly to external work is subject to and matches Carnot's T1-T2/T1 conversion limit as determined by the temperature of the reservoir from which the heat originated and the cycle's $\mathrm{T} 2$.

The T1-T2/T1 heat to work conversion rate of the heat input energy stored in all the molecular freedoms at each ascending temperature also matches Carnot efficiency because, by staging the heat input, that energy is converted to work during adiabatic expansion from $\mathrm{C}$ to $\mathrm{D}$ at the same instantaneous temperature that it entered the cycle. These two portions of the enthalpy change, the input energy that goes directly to external work and that which is stored by the freedoms can thus both be treated as having occurred isothermally and their rate of energy conversion from heat to work both match but can not exceed Carnot efficiency. This leaves the energy that goes to bond stretching. Though this energy would appear to be a wasted throughput, the volume changes that coincide with bond stretching and the resultant increases in the mean free path set up conditions for an energy transfer that negates the apparent inefficiency.

The amount of energy transferred from molecular deformation caused by collisions to molecular translation is determined by both the temperature at which the transfer occurs and the change in the molecule's mean free path associated with the change in volume occurring at that temperature. More energy gets transferred from deformation to translation during isobaric expansion from the heat input from each higher temperature reservoir than is transferred back during the same change in volume at the common T2. The temperature determines the amount of energy transferred per molecular collision while the changes in the mean free path caused by changes in volume determine the incidence of collision per unit of time.

Table I. $\mathrm{CO}_{2}$ state point data

\begin{tabular}{ccccc}
\hline $\begin{array}{c}\text { Temp. } \\
(\mathbf{K})\end{array}$ & $\begin{array}{c}\Delta \text { Density } \\
\left(\mathbf{k g} / \mathbf{m}^{\mathbf{3}}\right)\end{array}$ & $\begin{array}{c}\Delta \text { Enthalpy } \\
(\mathbf{k J} / \mathbf{k g})\end{array}$ & $\begin{array}{c}\Delta \text { Internal Energy } \\
(\mathbf{k J} / \mathbf{k g})\end{array}$ & $\begin{array}{c}\text { Bond Stretching } \\
(\mathbf{k J} / \mathbf{k g})\end{array}$ \\
\hline $270-275$ & 23.29 & 10.57 & 10.29 & 6.19 \\
$275-280$ & 24.63 & 10.83 & 10.51 & 6.36 \\
$280-285$ & 26.24 & 11.15 & 10.79 & 6.59 \\
$285-290$ & 28.20 & 11.55 & 11.14 & 6.94 \\
$290-295$ & 30.70 & 12.06 & 11.59 & 7.34 \\
$295-300$ & 33.94 & 12.73 & 12.17 & 7.89 \\
$300-305$ & 38.32 & 13.67 & 12.97 & 8.74 \\
$305-310$ & 44.30 & 14.96 & 14.07 & 9.82 \\
$310-315$ & 52.86 & 16.87 & 15.65 & 11.37 \\
$315-320$ & 64.78 & 19.68 & 17.92 & 13.62 \\
$320-325$ & 77.15 & 23.05 & 20.41 & 16.11 \\
$325-330$ & 78.51 & 24.72 & 21.16 & 16.81 \\
$330-335$ & 63.27 & 22.45 & 18.60 & 14.22 \\
$335-340$ & 45.49 & 18.63 & 15.03 & 10.63 \\
$340-345$ & 33.04 & 15.54 & 12.32 & 7.87 \\
$345-350$ & 25.05 & 13.37 & 10.48 & 6.00 \\
$350-355$ & 19.79 & 11.86 & 9.21 & 4.71 \\
$355-360$ & 16.17 & 10.76 & 8.31 & 3.83 \\
$360-365$ & 13.55 & 9.94 & 7.66 & 3.11 \\
$365-370$ & 11.60 & 9.30 & 7.14 & 2.56 \\
$370-375$ & 10.10 & 8.80 & 6.76 & 2.18 \\
$375-380$ & 8.91 & 8.37 & 6.44 & 1.82 \\
\hline & & & &
\end{tabular}

During the large isobaric changes in density and attendant large increases in the total heat input per degree between the temperatures of about $320 \mathrm{~K}$ and $335 \mathrm{~K}$ as demonstrated on the chart, there is per degree, a proportionately large increase in energy that goes to bond stretching. This increase in bond stretching energy coincides with a rapid increase in the mean free path in this range of volume change. This indicates that the reduced amount of time spent in collision verses translation produces a proportionately large shift of energy from deformation to translation. Because the Carnot efficiency of the input heat is calculated on a per degree basis, the 
larger T1-T2/T1 heat to work potential associated with these changes in temperature and volume coincide with the large shifts of energy from deformation to translation. Past this range of volume change the mean free path has become large and there is a rapid decrease in the rate of increase in the change of the mean free path per change in volume. This again coincides with the reduced energy requirement for bond stretching. Thus, the Carnot T1-T2/T1 efficiency of the heat input per degree matches the T1-T2/T1 work potential of the differential in the energy shifted from deformation to translational between the equivalent but opposite volume changes at the ascending $\mathrm{T} 1 \mathrm{~s}$ and the common $\mathrm{T} 2$.

The input energy that goes directly from heat to the translational freedom accomplishes the work of expansion from $\mathrm{B}$ to $\mathrm{C}$ while the energy stored in all the molecular freedoms with each increase in temperature goes to work during adiabatic expansion from $\mathrm{C}$ to $\mathrm{D}$. During these increases in volume the energy that goes to bond stretching is functioning in what could be likened to a free expansion were the transfer of the energy from being stored in deformation to translation negates its inefficiency by maintaining the molecular velocity at each change in temperature and volume during the associated increase in the molecule's mean free path.

The T1-T2/T1 Carnot efficiency work potential of the energy that is input to accomplish the bond stretching per degree is thus proportionate to the energy that is shifted from molecular deformation to translational during associated changes in the mean free path. When the Carnot efficiency of this energy shift is factored in with the Carnot efficiencies of the energy that goes directly to external work and to the molecular freedoms and then to work during adiabatic expansion, it yields the over all Q efficiency/T efficiency ratio of 1.00 .

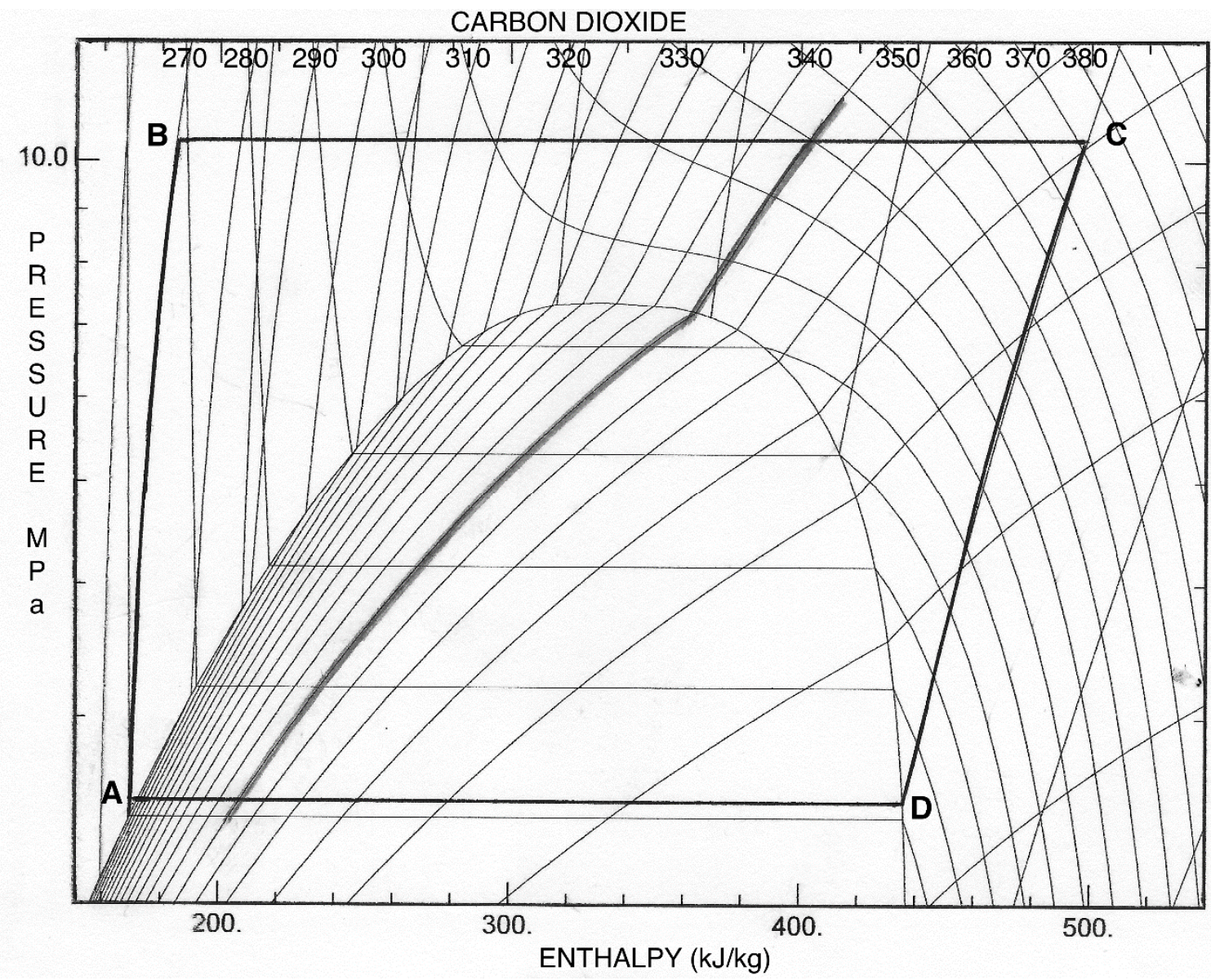

Figure 1. CO2 P-h diagram with shaded line of constant density and power cycle A-B-C-D-A

\section{Conclusion}

The points of this analysis are; first, acknowledging the fact that Carnot efficiency applies to any energy when it gets into the form of molecular translation and second, demonstrating the reason why the $\mathrm{Q} / \mathrm{T}$ ratio of 1.00 applies to all normal liquids operated in this cycle. This application of the second law then asserts that there is no 
possibility of transferring energy from the cycle's T2 to near its T1 by a differential in excess enthalpies of solution without elevating the $\mathrm{Q} / \mathrm{T}$ ratio above 1.00 . That energy transfer would produce a closed cyclical process that converted heat to work at a rate exceeding what is attainable with the ideal gas Carnot cycle.

The solvent density range where retrograde solubility occurs, as designated by the shaded constant density line on the diagram, is demonstrated by the 16 references cited in (Van Den Einde, 2016b). Solid solutes that yield positive excess enthalpies are, because of relatively weak solvent-solute bonds, the most likely solutes to experience retrograde solubility in the designated density range. This brings into question the original assumption made in the mid eighteen hundreds that the rate the ideal gas can convert heat to work between two absolute temperatures sets a universal limit on the convertibility of heat to work by a closed cyclical process.

\section{References}

National Institute of Standards and Technology, Boulder, CO (2007). REFPROP Version 8.

Van Den Einde, D. (2016a). A potential for complete Rankine cycle exhaust heat regeneration. Physics Essays, 29(3), 297-300.

Van Den Einde, D. (2016b). Solvent density effect on supercritical retrograde solubility. Physics Essays, 29(1), 110-112.

Van Den Einde, D. (2017). The effects of intermolecular energy transfers on power cycle efficiency. Physics Essays, 30(2), 224-225.

\section{Copyrights}

Copyright for this article is retained by the author(s), with first publication rights granted to the journal.

This is an open-access article distributed under the terms and conditions of the Creative Commons Attribution license (http://creativecommons.org/licenses/by/4.0/). 